\title{
MICROMECHANICAL ANALYSIS OF DETACHMENT MECHANSIMS OF SUFFUSION
}

\author{
QIRUI MA ${ }^{12}$, ANTOINE WAUTIER ${ }^{2}$ AND WEI ZHOU ${ }^{1}$ \\ ${ }^{1}$ School of Water Resources and Hydropower Engineering, Wuhan University \\ 8 Donghu South Road, 430072 Wuhan, China \\ ${ }^{2}$ INRAE, Aix-Marseille University, UR RECOVER, \\ 3275 Route Cézanne, CS 40061, Aix-en-Provence, France \\ *qirui.ma@inrae.fr
}

\begin{abstract}
Suffusion is a typical phenomenon of seepage-induced internal erosion, corresponding to fine particles erosion from the coarse matrix under the action of a fluid. A three-dimensional and spatially resolved fluid-solid coupling method based on lattice Boltzmann method (LBM) and discrete element method (DEM) is proposed to simulate suffusion in binary mixtures under anisotropic stress states. Based on such numerical simulations, eroded grains are identified and the mean squared displacement as well as the ratio between drag force and contact force of these eroded particles are computed to describe the detachment mechanism. The results show that the fluid force acting on eroded particles increases in the simulation which corresponding to the steep slope in squared displacements. Regardless of the initial state, the ratio between the fluid force and the contact force of the eroded particles displays a downward trend over time, reflecting the gradual dominance of the fluid force, which eventually leads to particle detachment and erosion. Based on the judgment between the direction of the fluid force and the direction of the contact force, a contact index $P$ is then proposed to determine whether the contact is going to slide or strengthen under the action of the fluid. The distribution of indexes $P$ for the contacts of eroded particles just prior to their detachment reflects that fluid induced sliding dominates. A particle detachment index is thus proposed and the overall detachment sensitivity of the binary samples is eventually investigated with respect to the fluid flow direction.
\end{abstract}

Key words: Granular Materials, Suffusion, DEM, LBM, Particle Detachment.

\section{INTRODUCTION}

Suffusion is a typical form of internal erosion, which can be described as the phenomenon of finest particle detach, transport and migrate in the void of structure formed by coarse particle. In geotechnical engineering, $46 \%$ of the earth rock dam damage is originated from internal erosion according to the recorded statistics ${ }^{[1][2]}$. Extensive experimental tests have been carried out to study internal stability and critical hydraulic gradient in internal erosion ${ }^{[3][4][5][6]}$. 
However, some research have found that the soil strength of corroded specimens is weakened ${ }^{[7]}$, but others have found that the corroded specimens have stronger liquefaction resistance under cyclic shear ${ }^{[8][9]}$. This means the influence of suffusion on soil has not been fully understood. However, due to the limitation of physical experiment conditions, micro mechanism cannot be well interpreted, so DEM (Discrete element method) provides a solution to simulate the suffusion from grain level ${ }^{[10]}$. Some researchers have already used unresolved CFD (computational fluid dynamic)-DEM coupling method to study the suffusion ${ }^{[11][12][13]}$. Compared with unresolved CFD-DEM, LBM (lattice Boltzmann method)-DEM coupling method, which can capture the details of fluid/grain interaction better, it is rare to carry out the research of suffusion. Moreover, many studies have not explored the process of particle detachment and clogging, but more focused on the final results. But the fully understanding of the particle detachment and migration in the microscale is essential to expound the occurrence of suffusion Therefore, in this paper, LBM-DEM method is used to simulate the mechanism of particle detachment in the suffusion process.

\section{SIMULATION PROCESS}

\subsection{Method}

The three-dimensional LBM-DEM coupling method based on two open-source software, LIGGGHTS responsible for the solid part in DEM ${ }^{[11]}$, Palabos solve the fluid region in LBM [14]. As for the fluid/g1 interaction between introduced respective In LBM, the governit
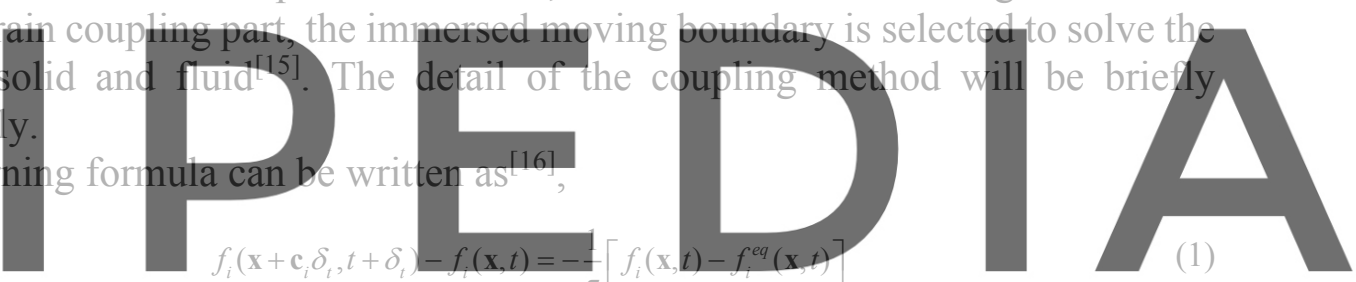

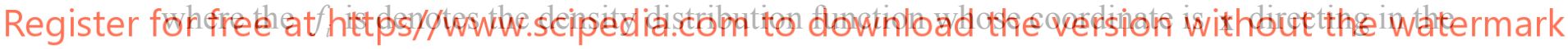

$i$-th path at time $t ; \mathbb{c}_{i}$ represents 19 discrete velocity vectors, because the D3Q19 LBM model

is used in this study; and $\tau$ is a relaxation coefficient, which controls the stability of LBM simulation. The equilibrium distribution functions $f_{i}^{\text {eq }}(\mathbf{x}, t)$ in the right side of Eq. (1) is defined as,

$$
f_{i}^{\text {eq }}=w_{i} \rho_{\text {fluid }}\left[1+\frac{\mathbf{c}_{i} \cdot \mathbf{u}_{\text {fluid }}}{c_{\mathrm{s}}{ }^{2}}+\frac{\left(\mathbf{c}_{i} \cdot \mathbf{u}_{\text {fluid }}\right)^{2}}{2 c_{\mathrm{s}}{ }^{4}}-\frac{\left|\mathbf{u}_{\text {fluid }}\right|^{2}}{2 c_{\mathrm{s}}{ }^{2}}\right] \quad(i=0, \ldots, 18)
$$

Where $w_{i}(i=0, \ldots, 18)$ are the weighting factors, $w_{0}=1 / 3, w_{1-6}=1 / 18, w_{7-18}=1 / 36 ; \rho_{\text {fluid }}$ and $\mathbf{u}_{\text {fluid }}$ are the density and velocity of fluid, respectively. The sound speed $c_{\mathrm{s}}=1 / 3$ in the lattice units in the 3D LBM model ${ }^{[17]}$.

According to the transfer method, the fluid velocity, fluid density, and fluid pressure in the macroscopic scale can be calculated through LBM quantities as follows ${ }^{[18][19]}$ :

$$
\rho_{\text {fluid }}=\sum_{i=0}^{18} f_{i}
$$




$$
\begin{gathered}
\mathbf{u}_{\text {fluid }}=\frac{1}{\rho_{\text {fluid }}} \sum_{i=0}^{18} \mathbf{c}_{i} f_{i} \\
p_{\text {fluid }}=c_{\mathrm{s}}^{2} \rho_{\text {fluid }}
\end{gathered}
$$

In DEM, the spherical particles' displacement is controlled by the Newton's second law of motion ${ }^{[10]}$ :

$$
\begin{aligned}
& m_{i} \mathbf{a}=\mathbf{F}_{\mathrm{c}}+\mathbf{F}_{\text {fluid }} \\
& I_{i} \dot{\mathbf{\omega}}=\mathbf{T}_{\mathrm{c}}+\mathbf{T}_{\text {fluid }}
\end{aligned}
$$

where $I_{i}$ is the moment of inertia of particle, $m_{i}$ is the mass of the particle; $\mathbf{a}$ is the motion acceleration; $\boldsymbol{\omega}$ is the angular velocity; $\mathbf{F}_{c}$ are the contact forces between particles and $\mathbf{T}_{\mathrm{c}}$ is corresponding torques provided by particle collision; $\mathbf{F}_{\text {fluid }}$ and $\mathbf{T}_{\text {fluid }}$ are the hydrodynamic force and the corresponding torque provided by the fluid part, respectively. The normal force $\mathbf{F}_{\mathrm{n}}$ and tangential force $\mathbf{F}_{\mathrm{t}}$ between two particles are calculated by the Hertz-Mindlin contact model.

Fluid-solid interaction calculations are achieved by adding a collision term $\Omega_{i}^{s}$ to Eq. (1), the govern equation becomes
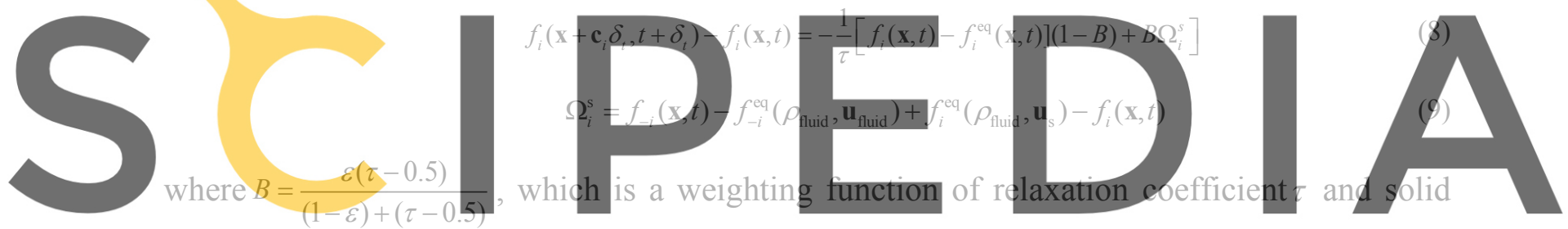

ratio $\varepsilon$ of the LBM cell. $\varepsilon=V_{\text {solid }} / V_{\text {cell }}$, and thus, $\varepsilon=0(1)$ yields $B=0(1)$. The subscript $-i$ in Register for free at https//www.scip.edia.com to download the version without the watermark the Eq. (9) represents the opposite direction of $i$.

Taking the effect of grain rotation into consideration, the velocity of the solid part $\mathbf{u}_{\mathrm{s}}$ is calculated as ${ }^{[20]}$,

$$
\mathbf{u}_{\mathrm{s}}=\mathbf{u}_{p}+\boldsymbol{\omega}_{p} \times\left[\left(\mathbf{x}+0.5 \mathbf{c}_{i} \delta_{t}\right)-\mathbf{x}_{p}\right]
$$

where $\mathbf{u}_{p}$ and $\boldsymbol{\omega}_{p}$ are translational and angular velocities of solid grain, and $\mathbf{x}_{p}$ is the position of solid grain.

Lastly, the hydrodynamic force $\mathbf{F}_{\text {fluid }}$ and the corresponding hydraulic torque $\mathbf{T}_{\text {fluid }}$ applied by fluid motion can be calculated as:

$$
\begin{gathered}
\mathbf{F}_{\text {fluid }}=\sum_{j=1}^{n} B_{j} \sum_{i=0}^{18} \Omega_{i}^{\mathrm{s}} \mathbf{c}_{i} \\
\mathbf{T}_{\text {fluid }}=\sum_{j=1}^{n}\left[B_{j}\left(\mathbf{x}_{j}-\mathbf{x}_{p}\right) \times \sum_{i=0}^{18} \Omega_{i}^{s} \mathbf{c}_{i}\right]
\end{gathered}
$$


Where $n$ is the total number of lattice cells covered by the solid part; $B_{j}$ refer to the weight coverage function in $j$-th lattice cell among $n$ lattice cells.

\subsection{Suffusion simulation}

The numerical simulation takes macroscopic fluid direction, hydraulic gradient, and vertical stress levels as factors to conduct research. The schematic figure of the simulation is shown in Fig. 1.

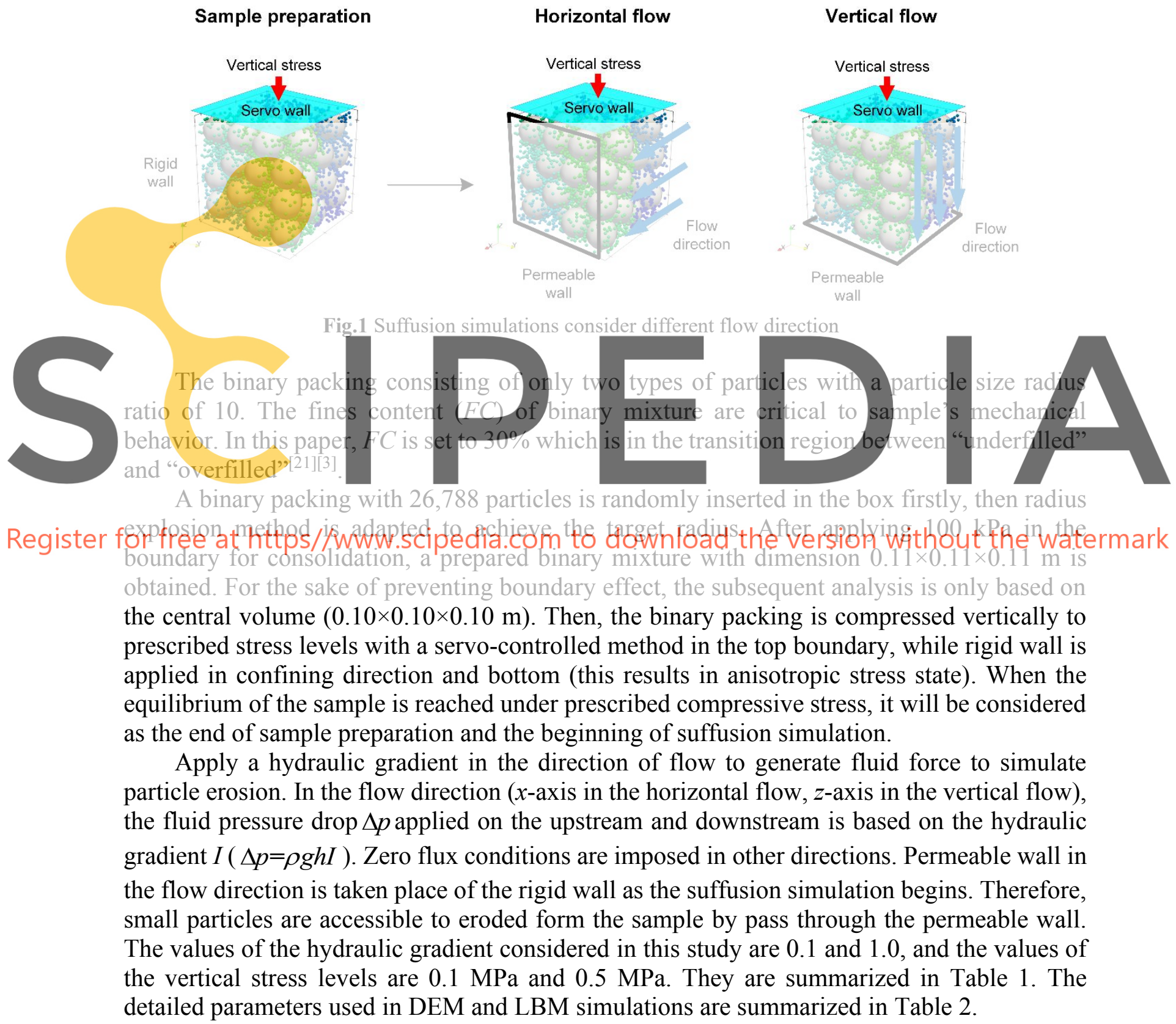


Table 1 The simulations carried out based on various conditions

\begin{tabular}{ccccc}
\hline Conditions & $\begin{array}{c}\text { Vertical stress, } \\
\sigma_{\mathrm{v}}(\mathrm{MPa})\end{array}$ & $\begin{array}{c}\text { Stress ratio, } \\
\eta\end{array}$ & $\begin{array}{c}\text { Hydraulic gradient, } \\
I\end{array}$ & Flow direction \\
\hline Case 1 & 0.1 & 0.7 & 1.0 & Horizontal \\
Case 2 & 0.5 & 1.1 & 0.1 & Horizontal \\
Case 3 & 0.5 & 1.1 & 1.0 & Horizontal \\
Case 4 & 0.5 & 1.1 & 1.0 & Vertical \\
Case 5 & 0.1 & 0.7 & - & - \\
Case 6 & 0.5 & 1.1 & - & - \\
\hline
\end{tabular}

Table 2 Parameters of the LBM-DEM simulations

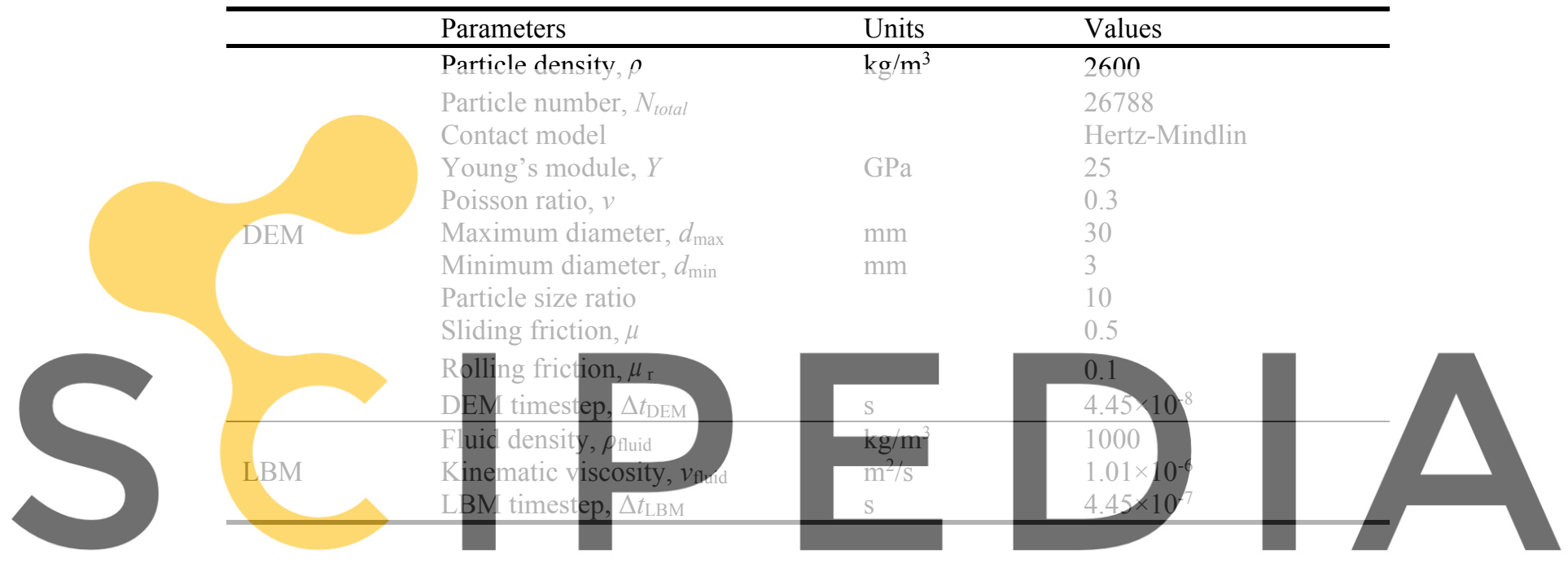

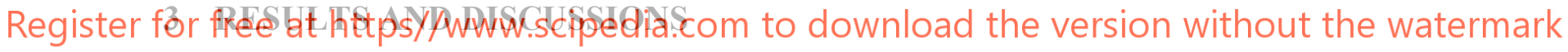

The fluid forces experienced by a few particular eroded particles during suffusion process are shown in Figure $2 \mathrm{a}$. In order to figure out the time dependence of displacement from the view of a single erosive particle, the variation of the translational mean squared displacement (TMSD) of these particles is also analyzed. The TMSD is calculated by the following equation:

$$
\left\langle d^{2}(t)\right\rangle=\left\langle\left|r_{j}(t)-r_{j}(0)\right|^{2}\right\rangle
$$

where $r_{j}(t)$ is the position of particle $j$ at time $t$. Figure $2 \mathrm{~b}$ describes the distribution of the TMSD in horizontal flow with vertical stress of $0.5 \mathrm{MPa}$. As shown in the Eqs. (14), if the eroded particles move at a steady speed, then the slope in the double logarithmic coordinate system of TMSD will be 2; and when it acts under a steady fluid force, the corresponding slope is 4 .

$$
\begin{aligned}
& \text { Constant } v: \text { Distance }=v \cdot t \quad \text { TMSD } \sim t^{2} \\
& \text { Constant } F: \text { Distance }=\frac{F}{2 m} t^{2} \quad \text { TMSD } \sim t^{4}
\end{aligned}
$$


(a)

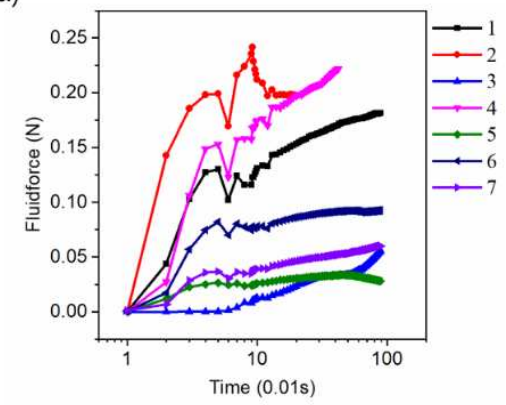

(b)

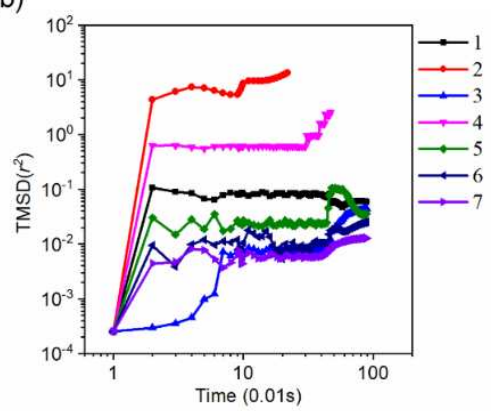

Fig. 2 (a) the variation of fluid force of eroded particles; (b) the TMSD of eroded particles; $(I=1.0$, $\sigma_{\mathrm{v}}=0.5 \mathrm{MPa}$, horizontal flow)

In Figure 2b, the initial slope for the TMSD is larger than 4 in the log-log plot which means that eroded particles move under the action of an increasing fluid force of eroded particles, which is confirmed in Figure 2a.

To describe the particle sensitivity to fluid forces, the ratio between the mean contact force and fluid foree of the eroded particles is judged by the following formula.
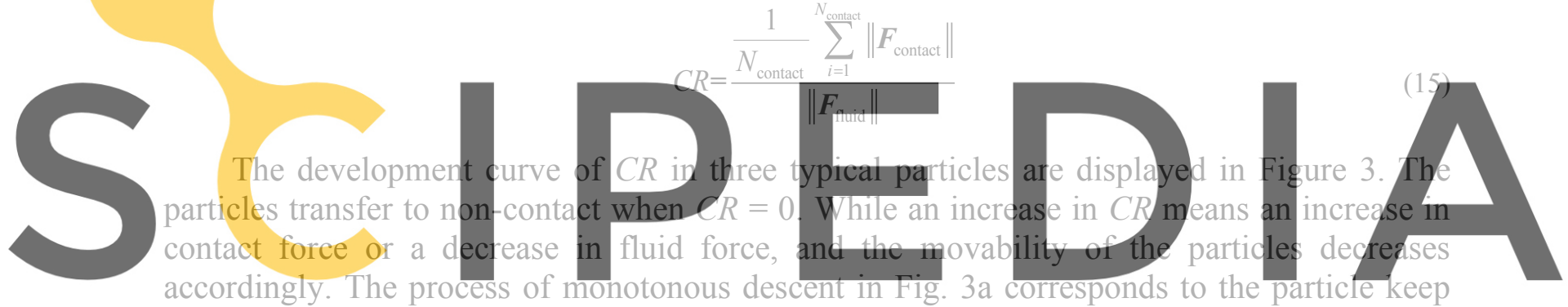

accordingly. The process of monotonous descent in Fig. 3a corresponds to the particle keep moving continuously without encountering local blockage, especially in the progression of the

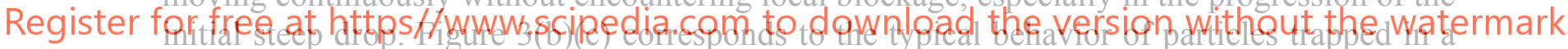

local pore structure, as the particles lose and gain contacts for several times. Overall, the general

decrease in $C R$ index in Figure 3 iliustrates that the fluid force gradualily dominates the competition with contact force and without any change in the stress boundary conditions, eroded particles do not get involved in the load-bearing skeleton anymore.

Based on the judgment between the direction of the fluid force and the direction of the contact force, a sliding index $P$ of each contact pair of eroded particles is then proposed to determine whether the contact is going to slide or strengthen under the action of the fluid. The index $P$ of a simple contact $i$ can be computed according to the formula below,

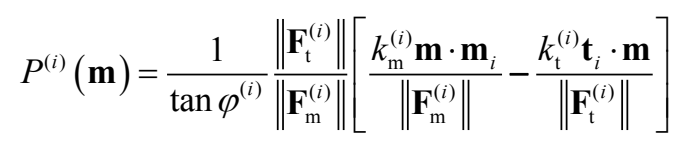

where $\mathbf{m}$ is the direction of fluid force of a particle, $\mathbf{m}_{i}$ and $\mathbf{t}_{i}$ are the normal and tangential vector of the $i$-th contact of the particle; $\mathbf{F}_{\mathrm{t}}^{(i)}$ and $\mathbf{F}_{\mathrm{m}}^{(i)}$ are the tangential force and contact force 
of $i$-th contact pair, $k_{\mathrm{m}}^{(i)}$ and $k_{\mathrm{t}}^{(i)}$ are the stiffness in the normal and tangential direction, $\varphi^{(i)}$ is the friction angle of $i$-th contact pair.

(a)

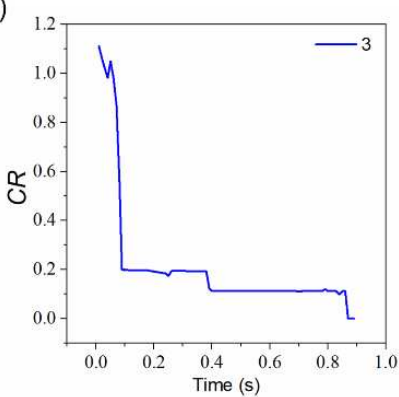

(b)

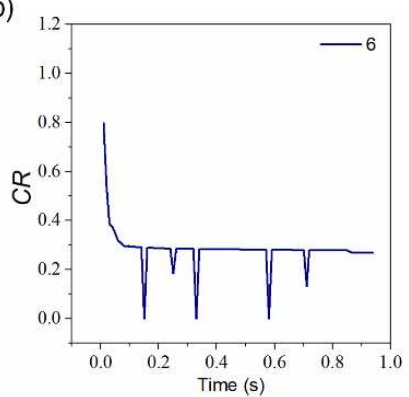

(c)

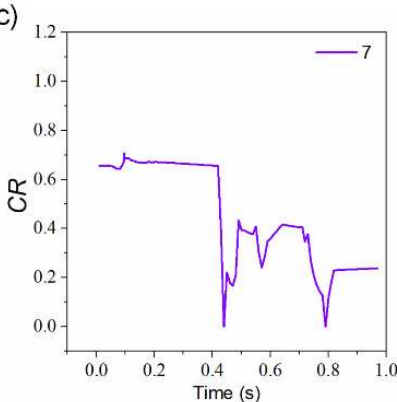

Fig. 3 Three typical $C R$ evolution curves of eroded particles

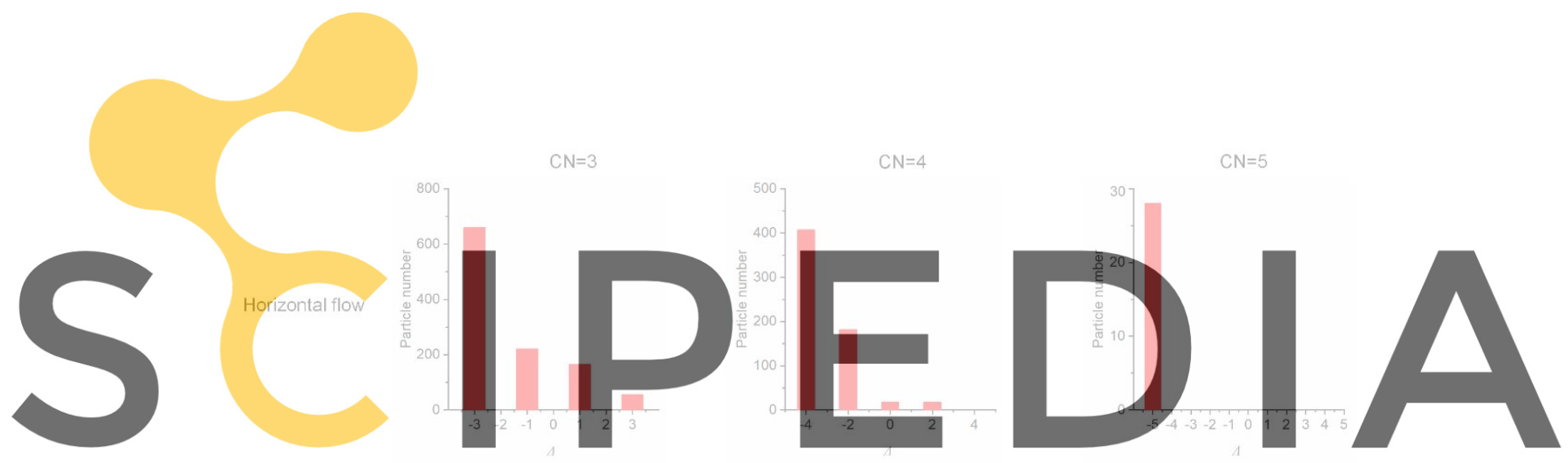

Register for free at https//www.scipedia.com to download the version without the watermark

Vertical flow

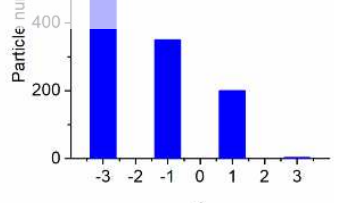

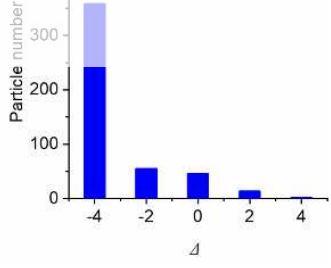

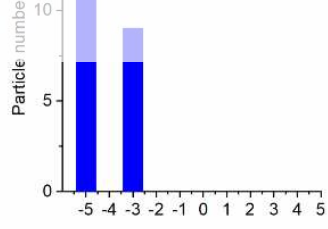

Fig.4 Distribution of $\Delta$ considering different $\mathrm{CN}$ and flow direction

According to the definition, $P<0$ reflects that the contact pair is going to slide and open if a fluid force in direction $\mathbf{m}$ is applied to the considered particle. $P^{(i)}$ index is attached to a given contact $i$. In order to construct a detachment index for a particle we define $\Delta=\operatorname{Number}\left(P^{(i)}(\mathbf{m})>0\right)-\operatorname{Number}\left(P^{(i)}(\mathbf{m})<0\right)$ which correspond to the difference between the number of contacts reinforced and weakened under the action of a fluid force in direction $\mathbf{m}$. As a result, a negative value for $\Delta$ is proposed to characterize the overall detachment sensitivity of a given particle subjected to a fluid force in direction $\mathbf{m}$. 
Figure 4 shows the $\Delta$ distribution for the eroded particles with different contact number (CN) at critical time (i.e. when the particles are about to lose contact and become free particles). The distribution of $\Delta$ for the contacts of eroded particles just prior to their detachment well reflects the sliding tendency of contact pairs, because of the large proportion of negative value. Detached particles are thus characterized by a set of contact forces which are unable to balance the additional force applied by the fluid without sliding or opening.

\section{CONCLUSIONS AND OUTLOOK}

This paper adopted a three-dimensional coupled LBM-DEM method to simulate the phenomenon of suffusion considering anisotropy stress state. The microscale detachment mechanism is investigated by proposed sliding index and contact force ratio in horizontal and vertical flow conditions. The main conclusions can be summarized as follows:

- $\quad$ The initial steep slope of mean squared displacement of eroded grains is observed, which attributed to the continuous increase of fluid force. Regardless of the initial state, the ratio between the fluid force and the contact force of the eroded particles displays a downward trend over time, reflecting the gradual dominance of the fluid force, which eventually leads to particle detachment and erosion.

Based on the judgment between the direction of the fluid force and the direction of the contact force, a contact sliding index $P$ is then proposed to determine whether the contact is going to slide or strengthen under the action of the fluid. The distribution of

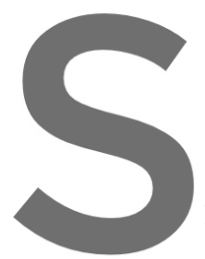
$P$ for the contact induced sliding The future work suffusion in different di from the perspective of the evolution of the sample; the fine partictes
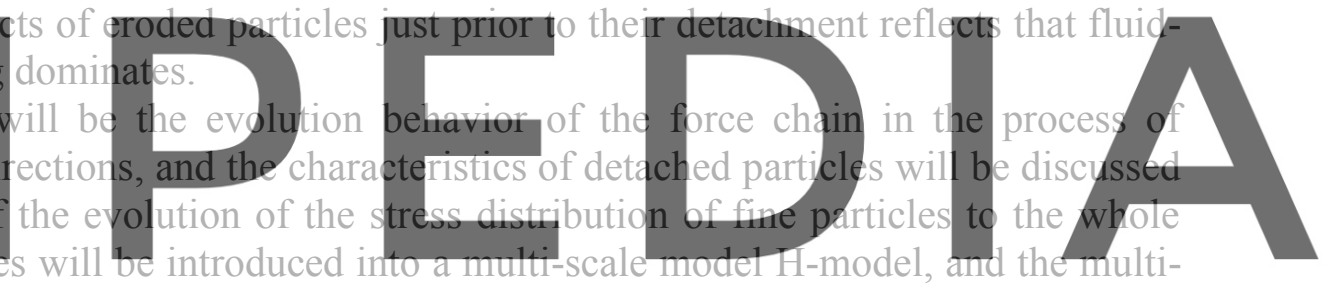

scale constitutive model considering internal erosion will established. This part of work is

Register farefrealatibttess//www.scipedia.com to download the version without the watermark

\section{REFERENCES}

[1] M. Foster, R. Fell, M. Spannagle, The statistics of embankment dam failures and accidents, Can. Geotech. J. 37 (2000) 1000-1024.

[2] M. Foster, R. Fell, M. Spannagle, A method for assessing the relative likelihood of failure of embankment dams by piping, Can. Geotech. J. (2000). doi:10.1139/t00-029.

[3] A.W. Skempton, J.M. Brogan, Experiments on piping in sandy gravels, Geotechnique. 44 (1994) 449460.

[4] M. Li, R.J. Fannin, Comparison of two criteria for internal stability of granular soil, Can. Geotech. J. 45 (2008) 1303-1309. doi:10.1139/T08-046.

[5] C. Chen, L.M. Zhang, D.S. Chang, Stress-Strain Behavior of Granular Soils Subjected to Internal Erosion, J. Geotech. Geoenvironmental Eng. (2016). doi:10.1061/(ASCE)GT.1943-5606.0001561.

[6] D. Marot, A. Rochim, H.H. Nguyen, F. Bendahmane, L. Sibille, Assessing the susceptibility of gapgraded soils to internal erosion: proposition of a new experimental methodology, Nat. Hazards. 83 (2016). doi:10.1007/s11069-016-2319-8.

[7] L. Ke, A. Takahashi, Experimental investigations on suffusion characteristics and its mechanical consequences on saturated cohesionless soil, Soils Found. (2014). doi:10.1016/j.sandf.2014.06.024.

[8] M. Xiao, N. Shwiyhat, Experimental investigation of the effects of suffusion on physical and geomechanic characteristics of sandy soils, Geotech. Test. J. (2012). doi:10.1520/GTJ104594. 
[9] A. Mehdizadeh, M.M. Disfani, R. Evans, A. Arulrajah, Impact of suffusion on the cyclic and post-cyclic behaviour of an internally unstable soil, Geotech. Lett. (2019). doi:10.1680/jgele.18.00128.

[10] P.A. Cundall, O.D.L. Strack, A discrete numerical model for granular assemblies, Geotechnique. (1979). doi:10.1680/geot.1979.29.1.47.

[11] C. Kloss, C. Goniva, A. Hager, S. Amberger, S. Pirker, Models, algorithms and validation for opensource DEM and CFD-DEM, Prog. Comput. Fluid Dyn. an Int. J. 12 (2012) 140-152.

[12] J. Tao, H. Tao, Factors affecting piping erosion resistance: Revisited with a numerical modeling approach, Int. J. Geomech. (2017). doi:10.1061/(ASCE)GM.1943-5622.0000999.

[13] K. Kawano, T. Shire, C.O. Sullivan, Coupled particle-fluid simulations of the initiation of suffusion Coupled particle-fluid simulations of the initiation of suffusion, (2018). doi:10.1016/j.sandf.2018.05.008.

[14] J. Latt, O. Malaspinas, D. Kontaxakis, A. Parmigiani, D. Lagrava, F. Brogi, M. Ben Belgacem, Y. Thorimbert, S. Leclaire, S. Li, F. Marson, J. Lemus, C. Kotsalos, R. Conradin, C. Coreixas, R. Petkantchin, F. Raynaud, J. Beny, B. Chopard, Palabos: Parallel Lattice Boltzmann Solver, Comput. Math. with Appl. (2020). doi:10.1016/j.camwa.2020.03.022.

[15] D.R. Noble, J.R. Torczynski, A lattice-boltzmann method for partially saturated computational cells, Int. J. Mod. Phys. C. 9 (1998) 1189-1201. doi:10.1142/S0129183198001084.

[16] P.L. Bhatnagar, E.P. Gross, M. Krook, A model for collision processes in gases. I. Small amplitude processes in charged and neutral one-component systems, Phys. Rev. (1954). doi:10.1103/PhysRev.94.511. C.K. Aidun, J.R. Clausen, Lattice-Boltzmann Method for Complex Flows, Annu. Rev. Fluid Mech. (2010). doi:10.1146/annurev-fluid-121108-145519.

[18] S. Harris, An introduction to the theory of the Boltzmann equation, Courier Corporation, 2004.

[19] R.L. Liboff, Kinetic theory: classical, quantum, and relativistic descriptions, Springer Science \& Business Media, 2003.

[20] D.R.J. Owen, C.R. Leonardi, Y.T. Feng, An efficient framework for fluid-structure interaction using the

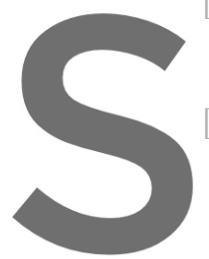
lattice Boltzmann me doi:10.1002/nme.298 T. Shire, C. O’Sulliv Unstable Soils, J. Ge doi:10.1061/(ASCE)GT.1943-5606.0001184.
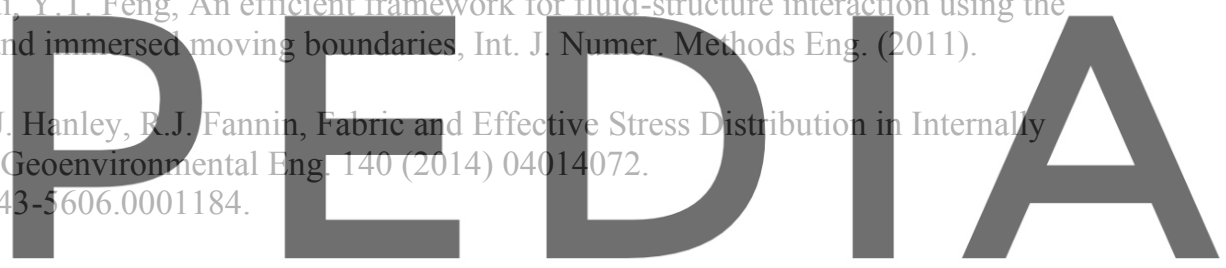